Classification

Physics Abstracts

$87.20-07.80-07.60 \mathrm{P}$

\title{
On the nature of the lipid monolayer phase transition
}

\author{
A. Fischer, M. Lösche, H. Möhwald and E. Sackmann \\ Physik Department E22 (Biophysics Group), Technische Universität München, D-8046 Garching, \\ F.R.G.
}

(Reçu le 9 avril 1984, accepté le 14 juin 1984)

\begin{abstract}
Résumé. - Nous présentons une technique améliorée pour l'étude par microscopie électronique et diffraction d'électrons de films monomoléculaires de lipide transférés d'une interface air/eau. Cette méthode, combinée avec une technique de microfluorescence, fournit un outil puissant pour étudier la microstructure des monocouches. Nous montrons que la pente non nulle qui apparaît dans les isothermes à la transition de phase fluide-cristalline provient de la coexistence de phases fluide et cristalline jusqu'à la transition de type second ordre vers l'état condensé complètement cristallin. Nous présentons un modèle électrostatique qui rend compte (1) de la stabilité thermodynamique dans la région de coexistence de l'état fluide et de l'état solide, (2) de la pente non nulle dans la région de transition, (3) de l'existence de plaquettes cristallines de taille uniforme à pression donnée au début de la région de transition.
\end{abstract}

\begin{abstract}
An improved technique to study transferred lipid monolayers by electron microscopy and electron diffraction is presented. In combination with a microfluorescence technique it provides a powerful technique to study the microstructure of monolayers. It is demonstrated that the nonhorizontal slope of the isotherms at the fluid-to-crystalline phase transition is due to the coexistence of fluid and crystalline phases up to the second order like transition to the completely condensed crystalline state. An electrostatic model is presented which explains (1) the thermodynamic stability of the fluid-solid coexistence, (2) the non-horizontal slope of the transition region, (3) the existence of a pressure dependent but uniform size of the crystalline platelets at the initial slope of the transition region.
\end{abstract}

A typical isotherm of a phospholipid monolayer is given in figure 1 for the case of dimyristoyl phosphatidic acid (DMPA). The pressure induced phase transition from the fluid to the crystalline state is not sharp but extends between a lower $\left(\pi_{\mathrm{m}}\right)$ and an upper transition pressure $\left(\pi_{\mathrm{c}}\right)$ while $\pi_{\mathrm{m}}-\pi_{\mathrm{c}}$ amounts to some $\mathrm{mN} / \mathrm{m}[1,2]$. The transition seems to be composed of two parts : slightly above $\pi_{\mathrm{m}}$ one observes a nearly straight line region of non-horizontal slope (called state II in Ref. [1]) which goes over into a steeper ascent (called state III in [1]) until the isotherm exhibits a break at $\pi_{c}$. The monolayer exhibits a fluid-like shear elasticity between $\pi_{\mathrm{m}}$ and $\pi_{\mathrm{c}}$ and is therefore also denoted as fluid condensed [3-5].

In the present paper clear evidence is provided that the monolayer exhibits a fluid-solid coexistence in the whole pressure region $\pi_{\mathrm{m}} \leqslant \pi \leqslant \pi_{\mathrm{c}}$. This coexistence is explained in terms of a different excess surface charge of the fluid and the crystalline phase and of electrostatic and steric 


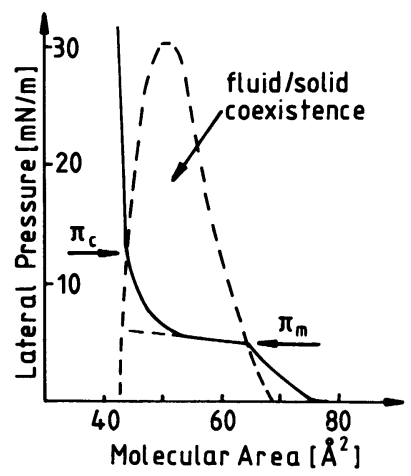

Fig. 1. - Pressure-area-diagram of $\mathrm{DMPA}^{-}$on pure water (pH 5.5) taken at a temperature of $30^{\circ} \mathrm{C}^{\text {accord- }}$ ing to [1]. The $\mathrm{pH}$ of 5.5 is mainly due to the presence of carbonate ions besides the counterions of $\mathrm{DMPA}^{-}$

interactions between the crystalline platelets. In particular we show that the electrostatic line energy at the fluid/solid interface can account for the mechanical stability of the coexisting phases.

In a recent paper we shoved that well defined monolayer states may be transferred from the air/water interface to very thin solid substrates and may thus be studied by conventional electron microscopy and electron diffraction. With this technique some evidence was provided that the non-horizontal slope of the isotherm is due to the coexistence of fluid and crystalline phases at least up to the transition pressure $\pi_{\mathrm{c}}$.

In the present note direct evidence for this is provided by combining an improved transfer technique with a modified method of electron microscopic observation. It is now possible to distinguish fluid and crystalline phases without any staining at low magnification. The mechanism for this contrast and the electronoptical configuration will be described elsewhere [6]. Simultaneously the molecular structure may be studied by electron diffraction. The monolayers are transferred to optimized Formvar-carbon-siliconmonoxid-grids. In combination with a recently developed microfluorescence technique [3] the present method provides a powerful tool to obtain detailed information about the microstructure of monolayers. The advantage of the fluorescence probe technique is that the monolayers may be directly observed on the water surface and that their dynamical properties can also be studied.

In the following, results for dimyristoylphosphatidic acid (DMPA) are presented : figure 2 shows fluorescence micrographs of a DMPA monolayer on pure water doped with DP-NBD-PE as fluorescence probe at various pressures above $\pi_{\mathrm{m}}$. The dark dendrite-like platelets are attributed to crystalline DMPA from which the fluorescence probe has been squeezed out into the fluid regions which appear bright. The size of the crystalline patches increases with increasing pressure (cf. Fig. 2) whereas the total number remains constant. The size and the distribution of the crystallites are fairly uniform and do not depend on time if the pressure is kept constant. The equilibrium size achieved may, however, depend on the rate of the pressure increase.

Figure 3 demonstrates that the DMPA monolayer may be transferred onto solid supports without a significant disturbance of the structure. Electron micrographs are given of a film transferred at a pressure (1) slightly above the beginning of the fluid-to-solid transition (Fig. 3a) (2) at the end of the straight-line region of the isotherm (Fig. 3b) and (3) slightly below the transition to the completely collapsed crystalline state at $\pi_{c}$ (Fig. $3 \mathrm{c}$ ). Dark and bright patches are clearly distinguished. The dark platelets grow with increasing pressure, start to coalesce and finally merge so that only a few bright patches are observed in figure $3 \mathrm{c}$. Figure $3 \mathrm{~d}$ exhibits an electron diffraction pattern of the dark platelet of figure 3c. Clearly, the dark patches correspond to a crystalline phase. Two hexagonal arrangements of diffraction spots with somewhat different 
a)

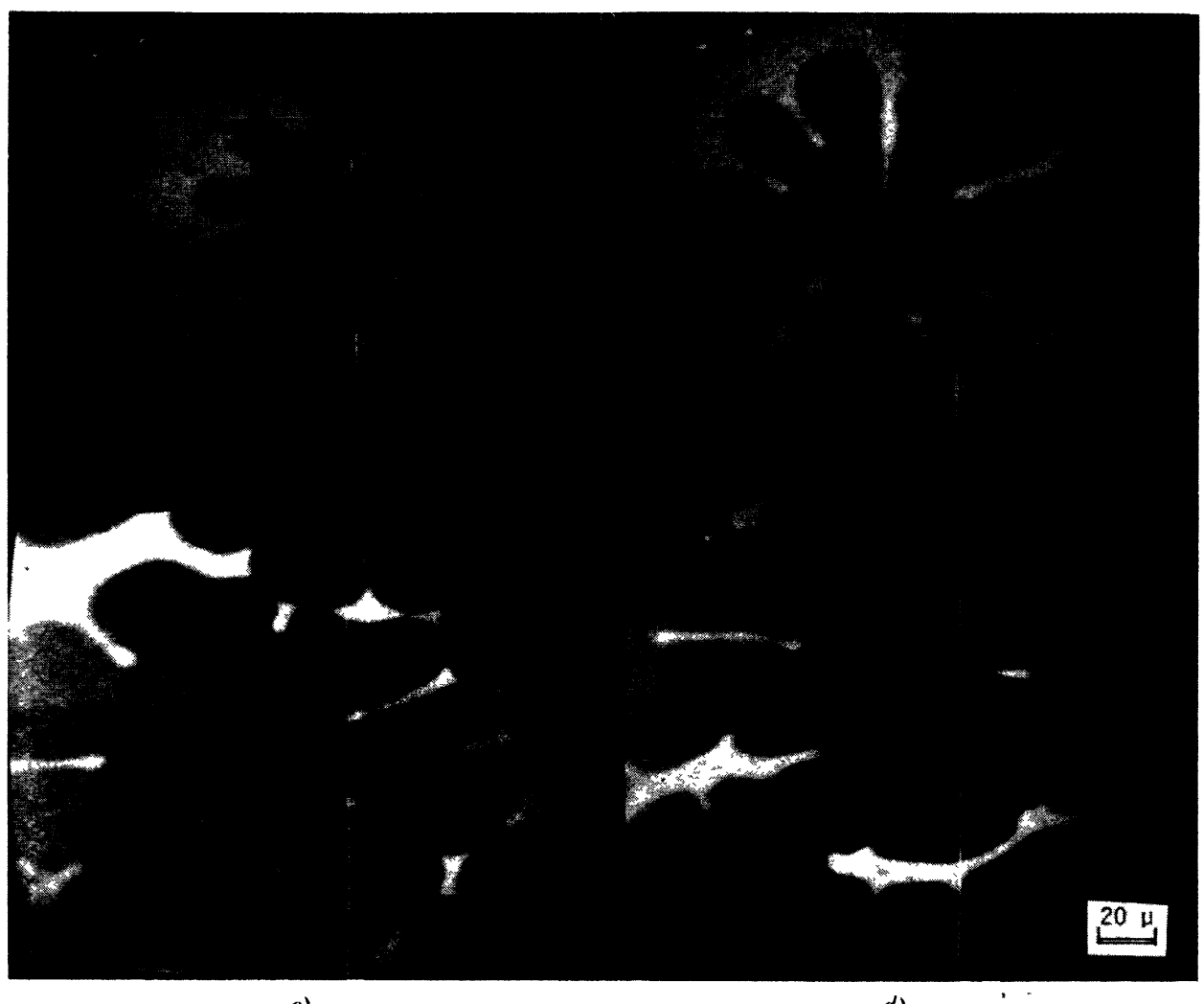

c) b)

d)

Fig. 2. - Fluorescence micrograph of DMPA monolayer for various pressures above $\pi_{\mathrm{m}}$. The concentration of the fluorescence probe L- $\alpha$-dipalmitoyl-nitrobenzoxadiazol-phosphatidyl-ethanolamine (DP-NBD-PE) is $0.3 \mathrm{~mol} \%$ with respect to DMPA a) and b) shows growth of crystalline platelet just above $\pi_{\mathrm{m}}$. c) and d) demonstrate how the platelets are merging at pressures near $\pi_{\mathrm{c}}$.

intensities are observed. This shows that the bright gap separates two crystallites with somewhat different orientation of the crystal planes. It should be noted that the dendrite-like form of the crystalline domains is a consequence of growth kinetics. They tend to be circular after some $30 \mathrm{~min}$. This indicates that the monolayer is in a quasi-stable equilibrium if the pressure is varied within the coexistence region.

In a separate experiment the previously described platinum shadowing technique was applied [2] in order to see whether there is a step in the thickness at the transition from the darker to the brighter regions. Within experimental error the two regions have the same thickness which demonstrates that the bright patches correspond to the fluid monolayer phase. Thus the small holes observed previously [2] may well have been formed by solidification of some of the fluid material during the film transfer. This was probably caused by the uranyl acetate used for staining. The diffraction of the bright areas is hidden below the diffuse ring of the Formvar substrate which shows that they correspond to a non-crystalline state.

Figures 2 and 3 provide evidence that the fluid-to-solid transition between $\pi_{\mathrm{m}}$ and $\pi_{\mathrm{c}}$ occurs in three steps : (1) the formation of crystalline platelets (nucleation), (2) their growth at increasing pressure and (3) the merging of two-dimensional crystallites. 

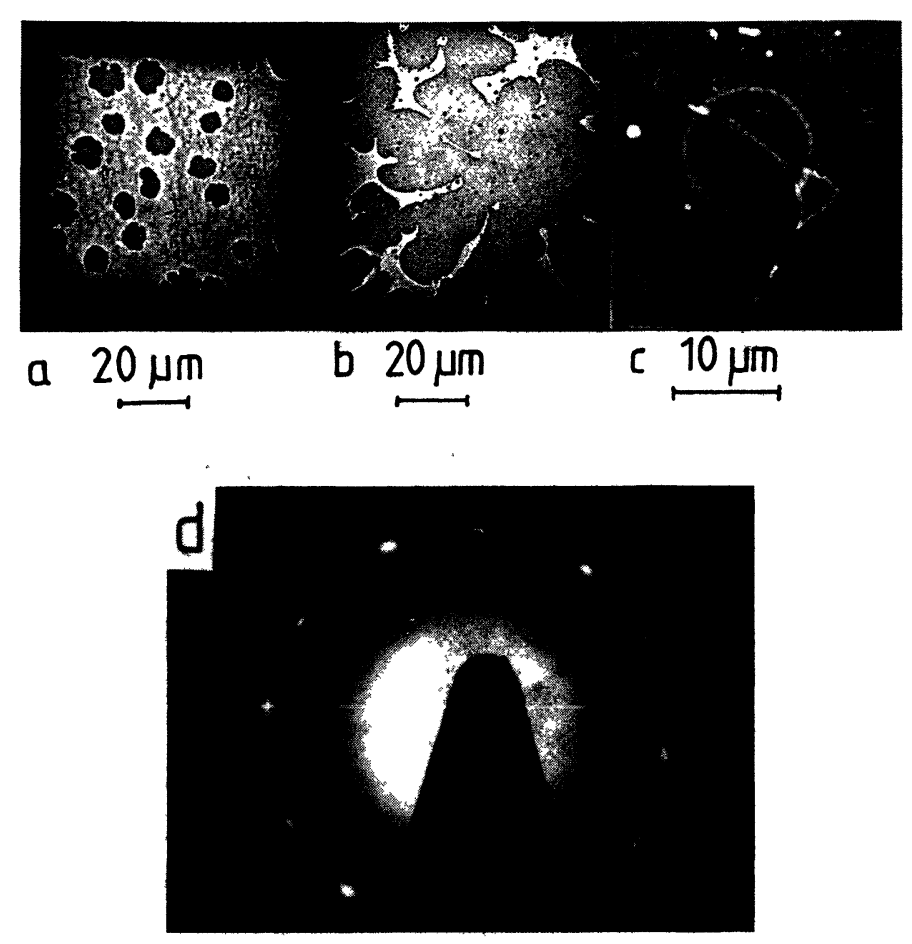

Fig. 3. - Electron micrograph of DMPA monolayers transferred at different pressures within the quasicontinuous transition region of the fluid-to-solid transition. The lateral pressure increases from a) to c). The dark spots are holes in the substrate. d) Electron diffraction pattern of monolayer in state just below $\pi_{\mathrm{c}}$ taken from dark area visible in figure $2 \mathrm{c}$. I he diffraction spots correspond to a lattice spacing of $d=4.2 \AA$.

From a thermodynamic point of view the coexistence of fluid and solid phases over a substantial pressure range can be attributed to the presence of ions in the aqueous phase. One is therefore dealing at least with a binary system so that the two-phase coexistence is consistent with the phase rule.

The second question is how mechanical equilibrium is established within the coexistence pressure region $\pi_{\mathrm{m}} \leqslant \pi \leqslant \pi_{\mathrm{c}}$. Obviously this could be accomplished by a line tension, $\gamma$, which arises at the boundary of the curved crystalline domains. This would lead to an increase in pressure within the fluid phase of the order of $\gamma / a$ (where $a$ is the domain radius; cf. Fig. 3) in the same way as the vapour pressure is larger over a curved liquid surface than over a flat one.

Both the formation of a strong line tension and the stabilization of a certain size of crystalline patches can be explained in terms of the electric field arising from the charging of the lipid water interface. As is well known, monolayers of both charged and zwitterionic lipids exhibit a surface potential $\psi$ [7]. This potential is associated with a surface charge density $\sigma$. Both $\psi$ and $\sigma$ increase with the lipid packing density so that the surface charge of the crystalline phase is higher than that of the fluid.

The electric field associated with the charging of the monolayer has two effects :

1) Due to the different surface charge densities of the fluid and solid phase an electrical potential difference $\Delta \psi$ arises at the fluid-to-solid interface. Obviously work must be done against $\Delta \psi$ in order to bring a molecule from the fluid into the solid phase. Thus a higher lateral pressure is necessary in order to crystallize all the lipid. 
2) The higher surface charge density of a solid platelet as compared to a fluid one leads to a repulsive force between the crystallites.

For the following discussion it is only necessary to consider the (mobile or fixed) excess charge densities at the monolayer surface which is different for the fluid $\left(\sigma_{\mathrm{f}}\right)$ and the solid phase $\left(\sigma_{\mathrm{c}}\right)$. Due to the low dielectric constant of the hydrocarbon chains $(\varepsilon \approx 1)$ the electric field decays slowly above the water level. Let $\psi_{\mathrm{f}}$ and $\psi_{\mathrm{c}}$ be the surface potentials of the fluid and the solid phase, respectively. The work per unit area associated with the creation of the excess electric field of the crystalline patches is then

$$
\Delta w=1 / 2 \Delta \psi \Delta \sigma
$$

where $\Delta \sigma=\sigma_{\mathrm{c}}-\sigma_{\mathrm{f}}$ and $\Delta \psi=\psi_{\mathrm{c}}-\psi_{\mathrm{f}}$. For a domain of radius $a$, the excess surface potential is approximately given by $\Delta \psi \sim \pi a \Delta \sigma$ and it follows

$$
\Delta w=\Delta \sigma^{2} a .
$$

The excess electric energy density of the crystalline domains thus increases linearly with their size. Consequently the lateral pressure must be increased in order to increase the domain size. This explains the non-horizontal slope within the straight line region of the isotherm as well as the fact that the domain size is constant and uniform at constant pressure.

Consider the case of $\mathrm{DMPA}^{-}$. At a $\mathrm{pH}$ of 5.5 this lipid carries one negative charge both in the fluid and in the solid phase. The packing density increases by about $20 \%$ at the fluid-to-solid transition which corresponds to an excess charge density of $\Delta \sigma=6 \times 10^{-6} \mathrm{Cb} . \mathrm{cm}^{-2}$. The difference in the surface potential $\Delta \psi=\psi_{\mathrm{c}}-\psi_{\mathrm{f}}$ is of the order of $100 \mathrm{mV}$ [7]. The excess surface energy is then $\Delta w \approx 1 \mathrm{erg} / \mathrm{cm}^{2} . \Delta w$ is indeed of the same order of magnitude as the work performed by the lateral pressure at the fluid-to-solid transition [1]. This work is given by the pressure difference between the onset of the transition (at $\pi_{\mathrm{m}}$ ) and the completion of the growth phase which corresponds to the end of the straight line region of the isotherm (cf. Fig. 1). The interfacial tension (force per unit length) at the fluid/solid interface can be expressed as $\gamma=q \Delta \psi / \sqrt{A}$ where $q$ is the charge and $A$ the area per lipid molecule. For a lipid carrying 0.2 elementary charge one obtains $\gamma \approx 2 \mathrm{mN} / \mathrm{m}$ which is again of the same order of magnitude as the pressure difference $\pi_{\mathrm{c}}-\pi_{\mathrm{m}}$. As the surface potential is reduced with increasing ionic strength one expects a corresponding decrease in the slope of the straight line region. This is indeed observed. Both the excess energy $\Delta w$ and the line tension $\gamma$ increase with increasing charge of the lipid molecules. According to the above considerations, the slope of the isotherm in the transition region should therefore increase

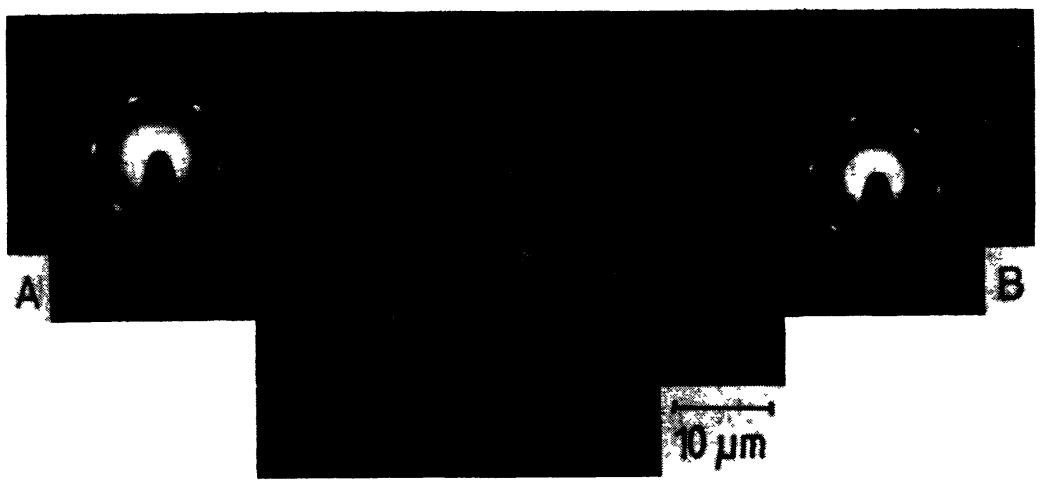

Fig. 4. - Comparison of electron diffraction taken from different parts (A, left; B, right) of merged platelet demonstrating that merging favours the formation of larger single crystals. 
with the lipid charge. This is in agreement with the previous finding that the slope and $\pi_{\mathrm{m}}-\pi_{\mathrm{c}}$ is larger for twofold charged DMPA than for the univalent lipid (cf. Ref. [1], Fig. 5).

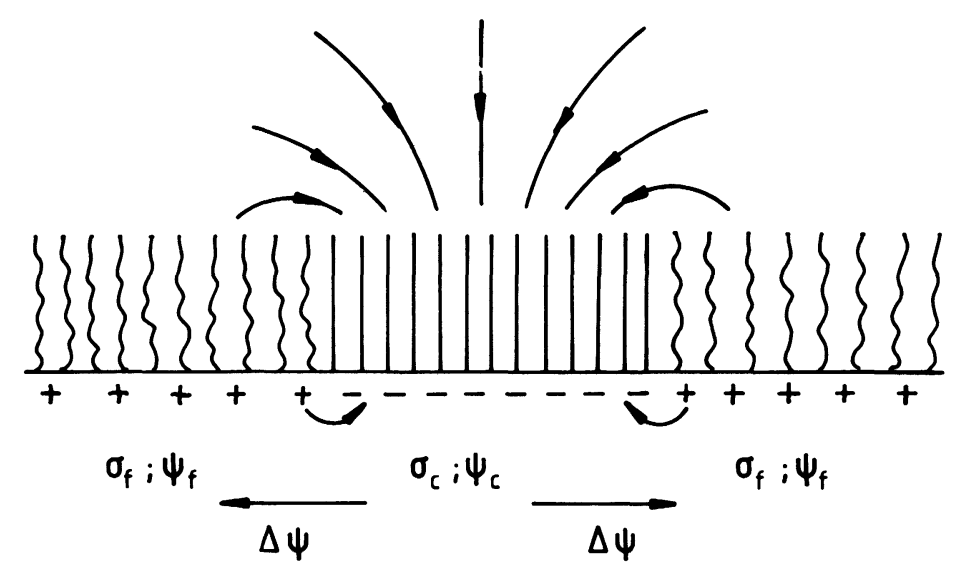

Fig. 5. - Schematic representation of excess charge distribution at lipid/water interface of fluid (surface charge density $\sigma_{\mathrm{f}}$, surface potential $\left.\psi_{\mathrm{f}}\right)$ and solid $\left(\sigma_{\mathrm{c}}, \psi_{\mathrm{c}}\right)$ patches. $\pi_{\mathrm{ex}}$ is the excess lateral pressure arising from the electric field built up at the fluid/solid interface.

The rather steep ascent of the isotherm above the straight line region of figure 1 can also be explained in terms of the excess charge of the crystalline domains which leads to their mutual repulsion. The interaction energy can be estimated according to

$$
E_{p}=p^{2} / d^{3}
$$

where $p$ is the total dipole moment of the platelets due to the excess surface charge which is assumed to be located in the centre of the domains. $d$ is the distance between the domains. For $\mathrm{DMPA}^{-}$and a value of $d=20 \mu \mathrm{m}$ one obtains $E_{p} \approx 1.3 \times 10^{-6} \mathrm{erg}$. The excess surface energy associated with this repulsion is $\Delta w_{\text {rep }}=E_{p} / a d=0.7 \mathrm{erg} . \mathrm{cm}^{-2}$ for $a=10 \mu \mathrm{m}$. Since $\Delta w_{\text {rep }}$ depends strongly on the distance $d$, the repulsion of the platelets may contribute considerably to the observed surface pressure at distances $d \leqslant 10 \mu \mathrm{m}$.

An alternative explanation for the steeper ascent of the isotherm above the straight line has been given previously [2]. It has been attributed to the fact that an additional pressure is necessary for the merging of the platelets. This process is expected to be associated with a shearing deformation of the platelets. The shear stress at $\pi_{\mathrm{c}}$ is of the order of $\tau=\left(\pi_{\mathrm{m}}-\pi_{\mathrm{c}}\right) \sqrt{A} / a$. $\tau$ is also a measure for the upper limit of the shear modulus, $G$, of the solid phase. For $\pi_{\mathrm{m}}-\pi_{\mathrm{c}}=10 \mathrm{mN} / \mathrm{m}, a=10^{-4} \mathrm{~cm}$ and $A=10^{-14} \mathrm{~cm}^{2}$ one obtains $G=10^{-2} \mathrm{mN} / \mathrm{m}$. It is interesting to note that this value is of the same size as value of $G$ observed for the erythrocyte membrane [8].

There are a number of theoretical models of the monolayer phase transition which try to explain the non-horizontal slope in terms of the dipole-dipole interaction of the head groups $[9,10]$. A non-horizontal slope could indeed be explained under the highly questionable assumption that the head group and the chain region are thermodynamically isolated [10]. The main drawback of such models is that they ignore the heterogeneity of the monolayer in the coexistence region.

Another interesting point is the following : if the crystalline domains are embedded in a fluid phase up to high pressures as is the case for mixtures, the charged platelets may eventually form a two-dimensional Wigner lattice. Such regular lattices have indeed been observed at high pressures 
(Lösche et al. [11]). Similar two-dimensional Wigner lattices have been observed for latex spheres on air/water interfaces $[12,13]$.

\section{References}

[1] Albrecht, O., Gruler, H. and Sackmann, E., J. Physique 39 (1978) 301.

[2] Fischer, A. and Sackmann, E., J. Physique 45 (1984) 517.

[3] Lösche, M., Sackmann, E. and Möhwald, H., Ber. Bunsenges. Phys. Chem. 87 (1983) 848.

[4] Cadenhead, D. A., Müller-Landau, F. and Kellner, B. M. J., in Ordering in Two-dimensions, S. K. Sinha, ed. (Elsevier North Holland ; Biomedical Press, Amsterdam) 1980, pp 73-81.

[5] Tscharner V., V. and McConnell, H. M., Biophys. J. 36 (1981) 56.

[6] Fischer, A., PhD-thesis, München 1984.

[7] Oldani, D., Hauser, H., Nichols, B. W. and Phillips, M. C., Biochim. Biophys. Acta 382 (1975) 1.

[8] Engelhardt, J., Gaub, H. and SackmanN, E., Nature 307 (1984) 378.

[9] Nagle, J. F., J. Membr. Biol. 27 (1976) 233.

[10] Baret, J. F., Progr. Surf. Membr. Sci. 14 (1981) 291.

[11] Lösche, M., Rabe, J., Fischer, A., Rucha, B. U., Knoll, W. and Möhwald, H., Thin Solid Films, in press.

Lösche, M. and MöHWALD, H., European Biophys. Journal, in press.

[12] Pieranski, P., Phys. Rev. Lett. 45 (1980) 569.

[13] Forsyth, Jr. P. A., Marcelja, S., Mitchell, D. J. and Ninham, B. W., Adv. Colloid Interface Sci. 9 (1978) 37. 